\title{
Pathological factors contributing to crossed cerebellar diaschisis in cerebral gliomas: a study combining perfusion, diffusion, and structural MR imaging
}

\author{
Xiaoxue Liu ${ }^{1}$. Jianrui $\mathrm{Li}^{1} \cdot$ Qiang Xu ${ }^{1} \cdot$ Dante Mantini ${ }^{2} \cdot$ Peng Wang $^{1} \cdot$ Yuan Xie ${ }^{1} \cdot$ Yifei Weng $^{1} \cdot$ Chiyuan Ma $^{3}$. \\ Kangjian Sun ${ }^{3} \cdot$ Zhiqiang Zhang ${ }^{1,4} \cdot$ Guangming Lu $^{1,4}$
}

Received: 14 November 2017 / Accepted: 26 February 2018 / Published online: 17 April 2018

(C) Springer-Verlag GmbH Germany, part of Springer Nature 2018

\begin{abstract}
Purpose To investigate imaging features of crossed cerebellar diaschisis (CCD) in cerebral gliomas, and its underlying pathophysiological mechanisms.

Methods Thirty-three pre-surgical patients with cerebral gliomas and 33 healthy controls underwent arterial spin-labeling, diffusion tensor imaging, and high-resolution T1-weighted imaging using MRI, in order to estimate cerebral blood flow $(\mathrm{CBF})$, white matter integrity, and lesion volume, respectively. Asymmetry indices of CBF in the cerebellum were used for evaluating the level of $C C D$ in the patients. These indices were correlated with clinical variables (lesion size and position, tumor histological grade, and CBF asymmetry) and diffusion tensor imaging parameters (fractional anisotropy and number of fibers in the cortico-ponto-cerebellar pathway and across the cerebral hemispheres), respectively.

Results The patients showed decreased CBF in the cerebellar hemisphere contralateral to the supratentorial tumor, and increased CBF asymmetry in the cerebellum (both $P<0.05$ ). CCD levels in high-grade gliomas were higher than those of low-grade gliomas $(P<0.05)$. CCD levels were negatively correlated with the size of the supratentorial lesions, and positively correlated with FA asymmetry in the cerebral fibers (both $P<0.05$ ).

Conclusions CCD in cerebral gliomas was specifically associated with tumor histological grade, lesion size, and white matter impairments in the hemisphere ipsilateral to the tumor. The findings implicated that observing CCD might have potential for assisting grading diagnosis of cerebral gliomas.
\end{abstract}

Keywords Crossed cerebellar diaschisis · Glioma · Magnetic resonance imaging · Perfusion imaging · Diffusion tensor imaging

\section{Abbreviation}

CCD crossed cerebellar diaschisis

Xiaoxue Liu and Jianrui Li contributed equally to this work.

Zhiqiang Zhang

zhangzq2001@126.com

$\checkmark$ Guangming Lu

cjr.luguangming@vip.163.com

1 Department of Medical Imaging, Jinling Hospital, Nanjing University School of Medicine, Nanjing 210002, China

2 Research Center for Motor Control and Neuroplasticity, KU Leuven, Leuven, Belgium

3 Department of Neurosurgery, Jinling Hospital, Nanjing University School of Medicine, Nanjing 210002, China

4 State Key Laboratory of Analytical Chemistry for Life Science, Nanjing University, Nanjing 210093, China

$\begin{array}{ll}\text { CPC } & \text { cortico-ponto-cerebellar } \\ \text { 3D ASL } & \text { three-dimensional arterial spin labeling } \\ \text { DTI } & \text { diffusion tensor imaging } \\ \text { CBF } & \text { cerebral blood flow } \\ \text { FA } & \text { fractional anisotropy } \\ \text { AI } & \text { asymmetry index }\end{array}$

\section{Introduction}

Crossed cerebellar diaschisis (CCD) is a phenomenon occurring in a variety of cerebral diseases with unilateral focal lesion, such as cerebral stroke [1-3], encephalitis [4, 5], epilepsy [6], and brain tumors [7, 8]. CCD is associated with depression of blood flow or metabolism in the cerebellar hemisphere contralateral to the supratentorial lesion on imaging measures $[9,10]$. The vast majority of CCD studies focused on cerebral 
stroke $[1-3,11-16]$. Studies using positron emission tomography (PET), single-photon emission computed tomography (SPECT), and arterial spin-labeling (ASL) imaging demonstrated hemodynamic changes, such as hypoperfusion and hypometabolism in the contralateral cerebellum. Moreover, a recent study combining PET with diffusion tensor imaging (DTI) suggested that interruption of the cortico-pontocerebellar (CPC) pathway might be a plausible mechanism underlying $\mathrm{CCD}[17,18]$.

CCD has been also demonstrated in cerebral gliomas in a limited number of reports [19]. Unlike other cerebral diseases with decreased CBF, cerebral gliomas with high grade may present elevated $\mathrm{CBF}$ and metabolism in the area around the lesion. A study using PET [19] found that the magnitude of CCD is mainly determined by tumor localization and size, and is also associated with tumor grade. Thus, it is of interest to study the interregional relationship of the blood flow between the cerebral lesion and the contralateral cerebellum. Moreover, evidence relating brain imaging features of CCD in cerebral glioma with features typically used in the clinics is yet lacking.

In the present study, we employed multi-modality MRI techniques, including arterial-spin-labeling (ASL)-based perfusion weighted imaging, diffusion tensor imaging (DTI), and structural imaging for an in-depth investigation of CCD in supratentorial cerebral gliomas. We aimed to better understand CCD in cerebral tumors, and specifically elucidate the mechanism of the interruption of the cortico-ponto-cerebellar pathway. To this end, we investigated (1) the clinical factors that correlate with brain imaging features of $\mathrm{CCD}$, and (2) the relationship between brain metabolism, structural integrity, and connectivity in supratentorial cerebral gliomas.

\section{Material and methods}

\section{Participants}

This study was approved by the local Medical Research Ethics Committee, and informed consent was obtained from all subjects or their legal representatives. Thirty-three patients (20 males and 13 females) with unilateral supratentorial gliomas were enrolled. The presence of gliomas and their characterization was confirmed by conventional MRI and pathological results. Patients with cerebellar abnormalities or bilateral cerebral brain lesions were excluded. Of these patients, 16 cases were high-grade gliomas (WHO 2016 CNS tumor classification guidelines: III-IV grades, including 13 glioblastomas, 2 anaplastic astrocytomas, 1 anaplastic oligoastrocytoma) and 17 cases were low-grade gliomas (WHO II grade, 14 diffuse astrocytomas, 1 oligodendroglioma, 2 oligoastrocytomas). The lesions of $18 / 15$ cases were located in the right/left hemisphere. Moreover, 33 healthy volunteers (14 males and 19 females) were recruited as controls. There were no differences in age and gender between patient and healthy control (HC) groups. The demographics and clinical information are shown in the Table 1.

\section{Data acquisition}

All subjects underwent an MR examination using a 3.0-T magnetic resonance (MR) scanner (Discovery MR750 System; GE Medical Systems, Milwaukee, WI, USA) with a 32-channel head coil. Three-dimensional T1-weighted imaging (3D-T1WI, BRAVO, TR/TE $=8200 / 3200 \mathrm{~ms}$, slice thickness $=4.0 \mathrm{~mm}, \mathrm{NEX}=1.0$ and number of slices $=36$ ) was acquired after intravenous body weight adapted administration of dimeglumine gadopentetate injection (Beijing Beilu Pharmaceutical Group, Beijing, China). Three-dimensional ASL data were acquired using pseudocontinuous labeling, background suppression, and a stack of spirals of 3D fast spin-echo imaging sequences. There were 512 sampling points on eight spirals, TR/TE $=5327 / 10.5 \mathrm{~ms}$, postlabel delay $(\mathrm{PLD})=2.5 \mathrm{~s}$, field of view $=24.0 \mathrm{~cm}$, slice thickness $=$ $4.0 \mathrm{~mm}$, number of slices $=36, \mathrm{NEX}=2.0$, and an acquisition time of $224 \mathrm{~s}$. DTI data were acquired using a standard diffusion-weighted spin-echo EPI pulse sequence with the following parameters: TR/TE $=5000 / 85.1 \mathrm{~ms}$, FOV $=$ $22.0 \mathrm{~cm}$, matrix $=128 \times 128$, slice thickness $=4.0 \mathrm{~mm}$, slice spacing $=0 \mathrm{~mm}$, bandwidth $=250 \mathrm{~Hz}, \mathrm{~b}$-value $=1000 \mathrm{~s} / \mathrm{mm}^{2}$, number of directions $=25$, acquisition time $=135 \mathrm{~s}$.

Moreover, routine structural images were also acquired for clinical diagnosis: axial T1-weighted imaging (FLAIR, TR/ $\mathrm{TE}=1750 / 24 \mathrm{~ms}$, slice thickness $=5.0 \mathrm{~mm}$, and slice spacing $=1.5 \mathrm{~mm}$ ), axial T2-weighted imaging (propeller, TR/ $\mathrm{TE}=5642 / 93 \mathrm{~ms}$, slice thickness $=5.0 \mathrm{~mm}$, and slice spacing $=1.5 \mathrm{~mm}$ ), and coronal $\mathrm{T} 2$ fluid-attenuated inversion recovery-weighted imaging $(\mathrm{TR} / \mathrm{TE}=9000 / 95 \mathrm{~ms}$, slices with thickness $=5.0 \mathrm{~mm}$, and slice spacing $=1.5 \mathrm{~mm}$ ).

\section{Data processing and analysis}

Images of 3D-ASL, DTI, and 3D-T1 were used for further analysis. Image preprocessing was conducted using SPM8 (www.fil.ion.ucl.ac.uk/spm). All images of the left glioma patients were left-to-right flipped for enlarging the sample size and allowing analysis of all patients as a homogeneous group. For 3D-ASL data, CBF maps were extracted from a GE AW4. 6 Workstation, and subsequently coregistered to individual structural 3D-T1 images. Each subject was normalized into the standard Montreal Neurological Institute (MNI) space with a 12-parameter affine-only nonlinear transformation. During process of normalization, cost-function modification was conducted to diminish the presence of lesion during the process. Lesion masks were manually and independently traced by 2 
Table 1 Demographics and clinical data of glioma patients and healthy controls

\begin{tabular}{llll}
\hline Protocols & Gliomas $(n=33)$ & HC $(n=33)$ & $P$ value \\
\hline Sex (M/F) & $20 / 13$ & $14 / 19$ & $0.139^{\mathrm{a}}$ \\
Age $( \pm \mathrm{SD})$, years & $49.94 \pm 14.12$ & $48.09 \pm 9.47$ & $0.535^{\mathrm{b}}$ \\
WHO grade (HGG/LGG) & $16 / 17$ & - & - \\
Lesion side (R/L) & $18 / 15$ & - & - \\
Flipped subjects' sex (M/F) & $8 / 7$ & $7 / 8$ & $0.715^{\mathrm{a}}$ \\
Flipped subjects' age ( $\pm \mathrm{SD})$, years & $48.80 \pm 12.32$ & $46.33 \pm 10.11$ & $0.554^{\mathrm{b}}$ \\
\hline
\end{tabular}

All of the above tests are performed using the software SPSS version 16.0 (SPSS Inc., Chicago, IL). A two-tailed $P$ value $<0.05$ was considered statistically significant

$H C$ healthy control, $H G G$ high-grade gliomas, $L G G$ low-grade gliomas

${ }^{\text {a }}$ The $P$ value for gender distribution in the two groups was obtained using the chi-squared test

${ }^{\mathrm{b}}$ The $P$ value for age between the two patient groups was obtained using a two sample $t$ test radiologists (L.X. and L.J.) in original space according to visible lesion (tumor +peritumoral edema and tumor only) areas on 3D-enhanced T1-weighted images, also guided by a T2weighted FLAIR image (Fig. 1). Finally, the normalized images were generated at a resolution of $3 \mathrm{~mm}$ isotropic, and then spatially smoothed with an 8-mm Gauss kernel.

We used the asymmetry index (AI) of CBF in the cerebellum to estimate the degree of CCD. AI was voxel-wisely calculated using the formula: $\mathrm{AI}=(\mathrm{A}-\mathrm{B}) /(\mathrm{A}+\mathrm{B})$, where $\mathrm{A}$ represents the $\mathrm{CBF}$ in the contralesional (i.e., contralateral to the cerebral tumor) cerebellum, $\mathrm{B}$ the $\mathrm{CBF}$ in ipsilesional cerebellum. In the healthy participants, $\mathrm{A}$ and $\mathrm{B}$ represent the $\mathrm{CBF}$ in the right and left cerebella, respectively. Negative values of the AI of CBF in the cerebellum indicate typical CCD. Moreover, lesion size was quantified in terms of the number of voxels within the lesion mask in each patient.

DTI images were processed using software of TrackVis (http://trackvis.org). After eddy current correction and brain extraction, fractional anisotropy (FA) maps were computed for all patients. Path tracing proceeded until either the FA was lower than 0.2 , or the angle between the current and the previous path segment was higher than 35. After whole-brain fiber tracking, short fibers lower than $20 \mathrm{~mm}$ and obvious false paths were discarded. The number of CPC fiber bundles and the average FA value were obtained by DTI tractography. We used a region of interest (ROI) approach to reconstruct CPC pathways and the fiber bundles in each cerebral hemisphere (Fig. 2). We delineated two ROIs on the FA maps for each tract. For the CPC tract, the seed region was set on the largest area of the cerebral hemisphere, and the target region was set on the largest area of the contralateral cerebellar hemisphere. For cerebral fiber bundles, the seed region was set in the side of the cerebral hemisphere with the largest area. Moreover, an avoidance region in the cerebral falx was set to avoid interference with contralateral fiber tracts.

\section{Statistical analysis}

\section{CCD estimation in the patients}

For estimating the degree of CCD, the CBF maps and the AI of $\mathrm{CBF}$ maps in the cerebellum were compared between the patients and healthy controls using voxel-wise two-sample $t$ tests, respectively. Moreover, a ROI-wise analysis was

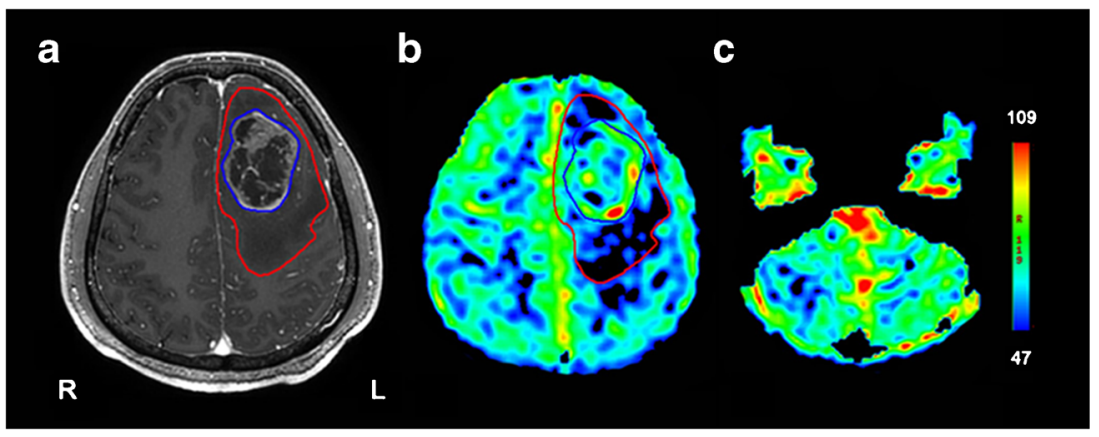

Fig. 1 Typical CCD presentation in a patient (male, 37 years) with a glioblastoma (WHO IV) in the left frontal lobe. a Contrast-enhanced T1-weighted image shows a glioma (area within blue circle) surrounded with edema (area between blue and red circles) in the left frontal lobe. b ASL perfusion map in a cerebral slice. The solid part of the tumor shows hyperperfusion; the cystic necrotic area and the peritumoral edema show hypoperfusion. $\mathbf{c}$ ASL perfusion map in a cerebellar slice. The cerebellar hemisphere contralateral to the cerebral tumor shows more hypoperfusion relative to the other hemisphere. ASL $=$ arterial-spin-labeling 


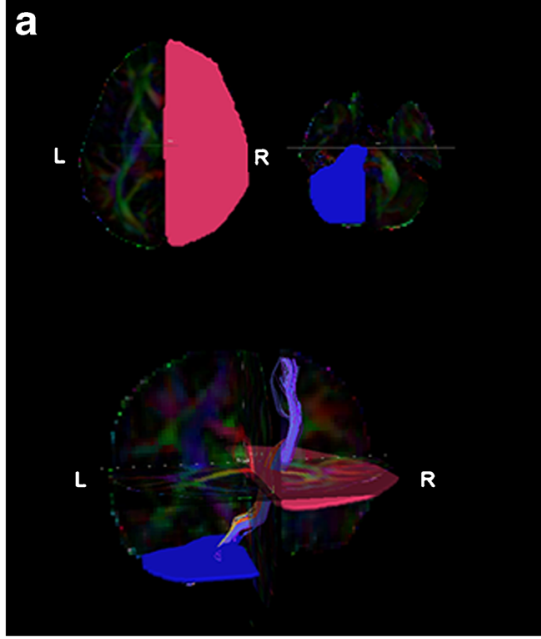

Fig. 2 DTI fiber tracking of CPC and unilateral cerebral fibers. a Fiber tracking of CPC. The seed region was set on the side of the cerebrum with the largest area, and the target region was set on the other side of the cerebellum with the largest area. b Fiber tracking of unilateral cerebral fibers. The seed region was set on the side of the cerebral hemisphere with

performed to identify the value of AI for the best discrimination between patients and controls.

\section{Relationships between CCD and pathological factors}

Four pathological factors were adopted. Firstly, to study whether the CCD were associated with the pathological grading of gliomas, the $\mathrm{AI}$ of $\mathrm{CBF}$ maps in the cerebellum were compared between the patients with high-grade gliomas (HGG) and those with low-grade gliomas (LGG) using twosample $t$ tests. Secondly, the AIs of CBF in the cerebellum were correlated with the cerebral lesion (tumor +peritumoral edema and tumor only) size. Thirdly, the AIs of CBF in the cerebellum were correlated with the AIs of CBF between the lesion and the same region in the opposite hemisphere. The second and third analyses were conducted within a regression model. The confounders of age and seizure occurrence were set as regressions in the analyses. Finally, the chi-square or Fisher exact test was used to observe the association between CCD and tumor location.

\section{Relationship between CCD and the pathology of white matter fibers}

We firstly examined the hemispheric difference of DTI parameters (FA and number of fiber bundles) in the CPC and cerebral fibers, respectively, using paired-sample $t$ tests. Furthermore, the AIs of DTI parameters were also calculated for CPC and cerebral fibers, respectively. For investigating the relationship between $\mathrm{CCD}$ and the integrity of white matter fibers, the $\mathrm{AI}$ of $\mathrm{CBF}$ in the cerebellum

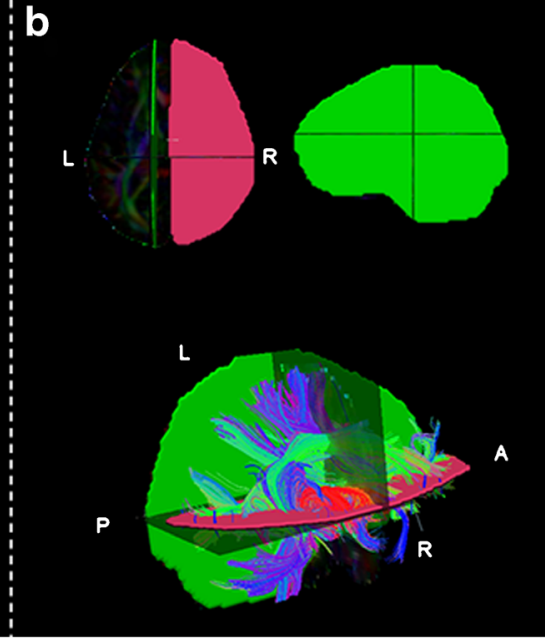

the largest area. Moreover, an avoidance region in the cerebral falx was set to avoid interference with contralateral fiber tracts. All ROIs were defined based on the FA maps. Abbreviations: $\mathrm{CPC}=$ cortico-pontocerebellar; ROI = region of interest; DTI = diffusion tensor imaging; $\mathrm{FA}=$ fractional anisotropy

was correlated with DTI parameters in the CPC and the cerebral fibers, respectively.

All of the above analyses were conducted at the voxel level using SPM8 $(P<0.05$, multiple correction with false discovery rate). The effect of age and gender was regressed out, by including these variables as covariates in the statistical analysis.

\section{Results}

\section{CCD in patients with glioma}

Compared with the healthy controls, the patients had significantly decreased $\mathrm{CBF}$ in the contralesional cerebellar hemisphere, and also showed decreased AIs of CBF in the cerebellum (both $P<0.05$ ). In the ROI analysis, the cutoff value of $\mathrm{AI}$ of $\mathrm{CBF}$ for best discriminating the patients $(-0.021 \pm 0.043)$ from healthy controls $(0.032 \pm 0.067)$ was -0.018 , with sensitivity and specificity of 84.85 and $57.58 \%$, respectively (Fig. 3 ).

\section{Association between CCD and other pathological factors}

The AIs of CBF in the cerebellum in high-grade gliomas were significantly lower than those of low-grade gliomas $(t=-2.547 P<0.05)$ (Fig. 4). The AIs of CBF in the cerebellum was negatively correlated with lesion (tumor + peritumoral edema) size $(r=-0.391 P<0.05)$ (Fig. 4) but not the tumor size only $(P>0.05)$, indicating that the increase in the lesion size leads to an increased asymmetry of $\mathrm{CBF}$ in 
Fig. 3 Voxel-wise comparisons of $\mathrm{CBF}$ and $\mathrm{AI}$ of $\mathrm{CBF}$ in the cerebellum between patients with glioma and healthy controls. a Comparison of $\mathrm{CBF}$ in the cerebellum. The patients show decreased CBF in the cerebellar hemisphere contralateral to the cerebral tumor. b Comparison of $\mathrm{AI}$ of CBF in the cerebellum. The patients show decreased AI of $\mathrm{CBF}$ in the cerebellum. The insert plots show individual values within the circles, for each of the two groups. **Presence of significant difference $(P<0.01)$. Abbreviations: $\mathrm{CBF}=$ cerebral blood flow; $\mathrm{AI}=$ asymmetry index; $\mathrm{HC}=$ healthy control

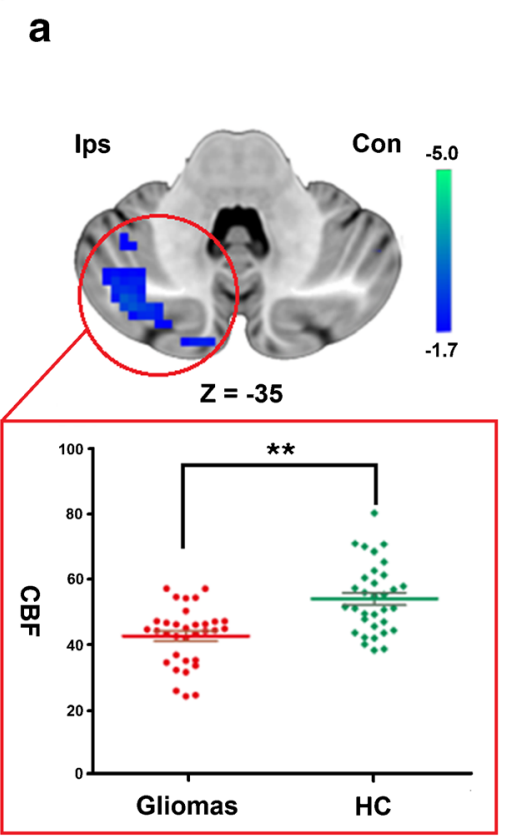

b

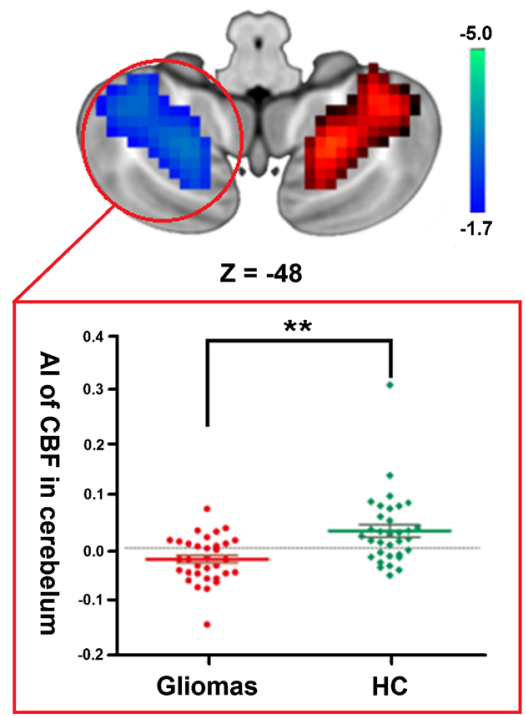

the cerebellum. The AIs of CBF in the cerebellum were not correlated with those of the supratentorial lesions (tumor + peritumoral edema) $(P>0.05)$. Moreover, there was no association between CCD and tumor position (all $P>0.05$ ) (Table 2).

\section{Relationship between CCD and white matter integrity}

For the cerebral fibers, there were differences of the FA (ipsilesional/contralesional $0.436 \pm 0.030 / 0.446 \pm 0.024 ; t=$ $-3.028, P<0.05$ ) and number of fiber bundles (ipsilesional/

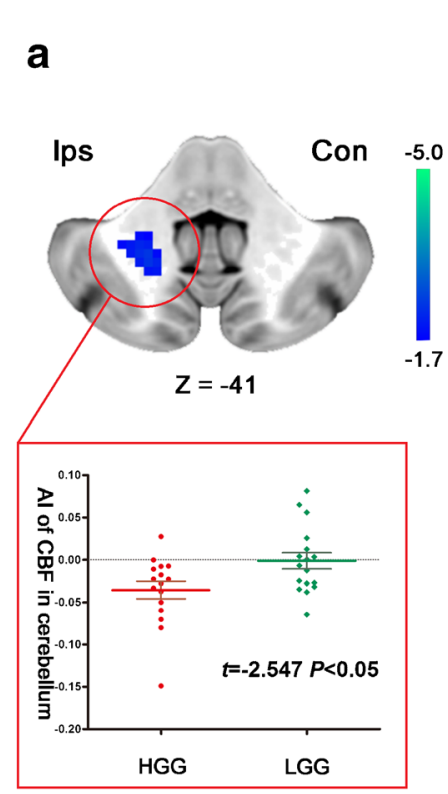

b

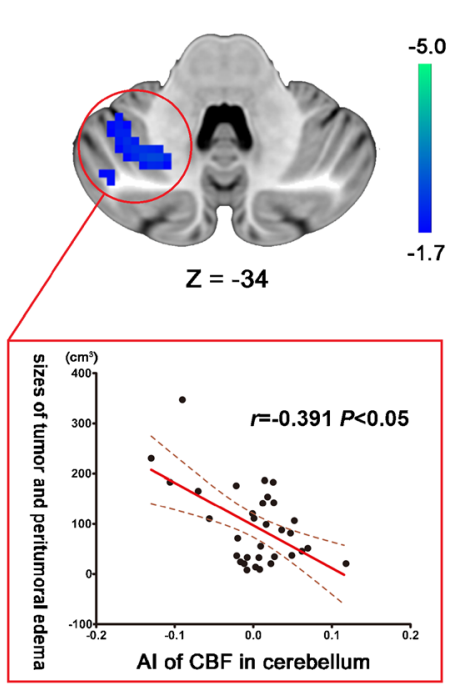

C

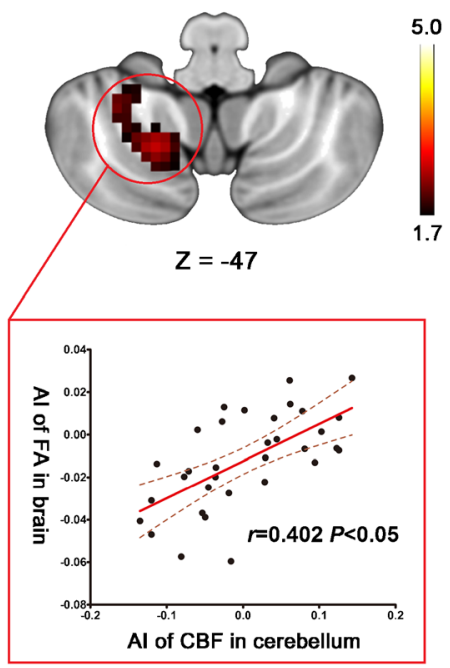

Fig. 4 Relationships between CCD and pathological factors and white matter integrity. a Two-sample $t$ test analysis shows that the AI of CBF in the cerebellum of the patients with HGGs is lower than those of LGGs. b Negative correlation between the AI of CBF in the cerebellum and lesion (tumor + peritumoral edema) size. c Positive correlations between the AI of $\mathrm{CBF}$ in the cerebellum and AI of FA of cerebral fiber bundles. Abbreviations: $\mathrm{CCD}=$ crossed cerebellar diaschisis; $\mathrm{CBF}=$ cerebral blood flow; $\mathrm{AI}=$ asymmetry index; $\mathrm{HGG}=$ high-grade gliomas; $\mathrm{LGG}=$ low-grade gliomas; $\mathrm{VOI}=$ lesion (tumor + peritumoral edema) size; FA = fractional anisotropy 
Table 2 Location of $\mathrm{CCD}(+)$ and $\mathrm{CCD}(-)$ gliomas

\begin{tabular}{lcclc}
\hline Location & Total sample $(n=52)$ & CCD + group $(n=28)$ & CCD - group $(n=24)$ & $P$ value $^{\mathrm{a}}$ \\
\hline Frontal lobe & $21(0.404)$ & $12(0.429)$ & $9(0.375)$ & 0.695 \\
Parietal lobe & $11(0.212)$ & $5(0.179)$ & $6(0.250)$ & 0.530 \\
Occipital lobe & $6(0.115)$ & $3(0.107)$ & $3(0.125)$ & 1.000 \\
Temporal lobe & $4(0.077)$ & $3(0.107)$ & $1(0.042)$ & 0.718 \\
Insular lobe & $2(0.038)$ & $2(0.071)$ & $0(0.000)$ & 0.493 \\
Basal ganglia & $4(0.077)$ & $1(0.036)$ & $3(0.125)$ & 0.324 \\
Corpus callosum & $4(0.077)$ & $2(0.071)$ & $2(0.083)$ & 1.000 \\
\hline
\end{tabular}

$C C D(+)$ crossed cerebellar diaschisis positive, $C C D(-)$ crossed cerebellar diaschisis negative

${ }^{\text {a }}$ The $P$ value for localization distribution was obtained using the chi-squared test contralesional $6473 \pm 1849 / 7330 \pm 2194 ; t=-3.387$, $P<0.05)$ between the two hemispheres. CPC tracking in the side of the tumor failed in 13 patients. Among the subjects with successful CPC tracking $(n=20)$, there were differences in FA values (ipsilesional/contralesional $0.307 \pm 0.252 / 0.467$ $\pm 0.153 ; t=-2.854, P<0.05$ ) and number of fiber bundles (ipsilesional/contralesional $22 \pm 46 / 59 \pm 68 ; t=-2.434$, $P<0.05$ ) between bilateral CPCs (Fig. 5).

There were significant positive correlations between AIs of $\mathrm{CBF}$ in the cerebellum and AIs of FA of the cerebral fibers $(r=0.402, P<0.05)$ (Fig. 4), but no correlation was found between AIs of $\mathrm{CBF}$ and the other DTI measures (all $P>0.05)$.

\section{Discussion}

This study used ASL-based perfusion MRI to estimate the CCD phenomenon in cerebral gliomas. The observed decrease of the $\mathrm{CBF}$ in the contralateral cerebellum and the elevated asymmetry of the cerebellar $\mathrm{CBF}$ is indicative of this phenomenon. CCD in cerebral gliomas was associated with the pathological grade and lesion (tumor + peritumoral edema) size. Moreover, by using DTI data, we further found that CCD was correlated with the AIs of the FA of cerebral fiber bundles.
This is the first study that combined ASL-based perfusion and diffusion tensor MR imaging to investigate the CCD phenomenon in patients with glioma. We found that patients with glioma showed decreased $\mathrm{CBF}$ in the contralateral cerebellum and elevated asymmetry of cerebellar CBF. The finding is concordant with the previous findings obtained using PET [19], ASL, and SPECT techniques [20].

We also found that the CCD levels were associated with the pathological grade and lesion (tumor + peritumoral edema) size, but not with the tumor size only. A previous study [19] using PET found that CCD levels in the patients with HGGs were higher than those of the LGGs, but CCD levels were not correlated with tumor size. Those findings support the concept that a highgrade tumor, with stronger invasiveness and more severe damage to the brain, is more likely to cause CCD. Some studies on other brain diseases also point out that CCD is related to the volume of the lesion [21-23]. We further indicated that peritumoral edema should be considered when evaluating the causes of CCD.

We found that the $\mathrm{AI}$ of $\mathrm{CBF}$ in the cerebellum (i.e., the CCD level) was not correlated with that of the supratentorial lesions. This is not consistent with the finding in cerebral stroke [24]. In ischemic strokes, the infarcted cerebral region commonly shows hypoperfusion;

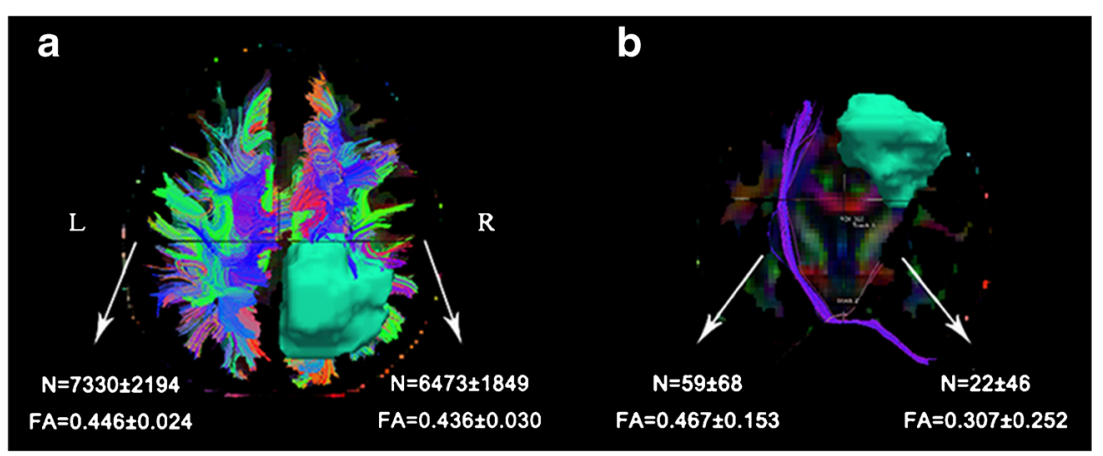

Fig. 5 DTI tractography for the fiber bundles in the cerebrum (a) and CPC (b) in the patients. The figure is an example of patient with a glioma in the parietal cortex. The plots show hemispheric differences of the FA

values and number of fibers in the cerebrum and $\mathrm{CPC}$ in the patient group. Abbreviations: $\mathrm{CPC}=$ cortico-ponto-cerebellar; DTI $=$ diffusion tensor imaging; $\mathrm{FA}=$ fractional anisotropy 
on the contrary, high-grade tumors may show hyperperfusion in the solid area and hypoperfuison in the cystic potion and peritumoral edema, which might impact on the $\mathrm{AI}$ of $\mathrm{CBF}$ in the lesioned area. Moreover, we did not find an association between CCD occurrence and location of tumors, unlike previous studies [19]. The heterogeneity of the patients' data or of the analysis methods used might be the cause of this inconsistence.

We investigated for the first time the relationship between CCD and pathological changes of the white matter fibers in cerebral gliomas. We found that the DTI parameters in the ipsilateral CPC in the lesional side were altered, suggesting an impairment of the $\mathrm{CPC}$ associated with tumor invasiveness. A similar imaging result has been reported in a stroke study [25]. CCD has been interpreted as metabolic effect caused by neuronal differentiation in the impaired CPC pathway $[25,26]$. We also found that the CCD levels were correlated with alterations of FA values in the hemispheric fibers. This result suggested that impairment of white matter integrity in the cerebrum also contributes to CCD. These results point out a relationship between perfusion and diffusion, and are of great significance for further exploring the pathological mechanism in the cerebral gliomas. Moreover, a follow-up study to investigate the possible plastic mechanism of CCD is significant.

Several limitations of the study should be mentioned. Firstly, we performed a left-to-right flipping of the MR images for enlarging the sample size, which inherently does not permit to investigate brain lateralization. Secondly, the homogeneity of pathological types of gliomas included in the study might affect the results. Thirdly, we assessed the possible associations between a limited number of clinical factors in cerebral gliomas and $\mathrm{CCD}$. Future studies are warranted to investigate the relationship between CCD in cerebral glioma and behavioral performance, especially in relation to motor and cognitive functions. Finally, it should be considered that brain imaging measures are dependent on the analysis approach used and we made specific methodological choices for enhancing the reliability of our results. For example, we used deterministic tractography in DTI data analysis and cost-function masking in perfusion data analysis. However, other analysis approaches may be also explored in the future.

In conclusion, by combining structural, perfusion, and diffusion MRI, we investigated the CCD phenomenon in cerebral glioma. We found that CCD was correlated with tumor histological grade, lesion (tumor + peritumoral edema) size, and importantly, with white matter fiber impairments in the hemisphere ipsilateral to the tumor. These results support the idea that tumors may impair local neuronal functioning and may also cause disruption of nerve connections, leading to CCD. The study provided novel insights into the clinical and pathological mechanisms of CCD in human cerebral gliomas, and implicated that observing CCD might have potential for assisting grading diagnosis of cerebral gliomas.

\section{Compliance with ethical standards}

Funding This study was funded by the Natural Science Foundation of China (Grant no. 81530054), the Chinese Post-Doctor Foundation (2016M603064), the Post-Doctor Foundation Jiangsu province (1501169B), Key Talents Project of Jiangsu Province (Grant nos. ZDRCA2016093), Independent Research Project in State Key Laboratory of Analytical Chemistry for Life Science (Grant nos. 5431ZZXM1716) and the Wellcome Trust (Grant no. 101253/Z/13/Z).

Conflict of interest The authors declare that they have no conflict of interest.

Ethical approval All procedures performed in studies involving human participants were in accordance with the ethical standards of the institutional and/or national research committee and with the 1964 Helsinki declaration and its later amendments or comparable ethical standards.

Informed consent Informed consent was obtained from all individual participants included in the study.

\section{References}

1. Lin D, Kleinman J, Wityk R et al (2009) Crossed cerebellar diaschisis in acute stroke detected by dynamic susceptibility contrast MR perfusion imaging. AJNR Am J Neuroradiol 30:710-715

2. Szilágyi G, Vas A, Kerényi L, Nagy Z, Csiba L, Gulyás B (2012) Correlation between crossed cerebellar diaschisis and clinical neurological scales. Acta Neurol Scand 125:373-381

3. Jeon YW, Kim SH, Lee JY, Whang K, Kim MS, Kim YJ, Lee MS, Brain Research Group (2012) Dynamic CT perfusion imaging for the detection of crossed cerebellar diaschisis in acute ischemic stroke. Korean J Radiol 13:12-19

4. Mahale R, Mehta A, Rangasetty S (2015) Crossed cerebellar diaschisis due to Rasmussen encephalitis. Pediatr Neurol 53: 272-273

5. Cianfoni A, Luigetti M, Bradshaw ML, Welsh CT, Edwards J, Glazier S (2010) MRI findings of crossed cerebellar diaschisis in a case of Rasmussen's encephalitis. J Neurol 257:1748-1750

6. Ohe Y, Hayashi T, Deguchi I et al (2013) A case of nonconvulsive status epilepticus with a reversible contralateral cerebellar lesion: temporal changes in magnetic resonance imaging and singlephoton emission computed tomography finding. J Stroke Cerebrovasc Dis 22:e639-e642

7. Teoh EJ, Green AL, Bradley KM (2014) Crossed cerebellar diaschisis due to cerebral diffuse large $\mathrm{B}$ cell lymphoma on $18 \mathrm{~F}$ FDG PET/CT. Int J Hematol 100:415-416

8. Calabria F, Schillaci O (2012) Recurrent glioma and crossed cerebellar diaschisis in a patient examined with $18 \mathrm{~F}-\mathrm{DOPA}$ and $18 \mathrm{~F}-$ FDG PET/CT. Clin Nucl Med 37:878-879

9. Feeney DM, Baron JC (1986) Diaschisis. Stroke 17:817-830

10. Baron JC, Bousser MG, Comar D, Castaigne P (1981) "Crossed cerebellar diaschisis" in human supratentorial brain infarction. Trans Am Neurol Assoc 105:459-461 
11. Kamouchi M, Fujishima M, Saku Y, Ibayashi S, Iida M (2004) Crossed cerebellar hypoperfusion in hyperacute ischemic stroke. J Neurol Sci 225:65-69

12. Serteser M, Ozben T, Gümüslü S, Balkan S, Balkan E (2001) Biochemical evidence of crossed cerebellar diaschisis in terms of nitric oxide indicators and lipid peroxidation products in rats during focal cerebral ischemia. Acta Neurol Scand 103:43-48

13. Komaba Y, Mishina M, Utsumi K, Katayama Y, Kobayashi S, Mori O (2004) Crossed cerebellar diaschisis in patients with cortical infarction logistic regression analysis to control for confounding effects. Stroke 35:472-476

14. Madai VI, Altaner A, Stengl KL, Zaro-Weber O, Heiss WD, von Samson-Himmelstjerna FC, Sobesky J (2011) Crossed cerebellar diaschisis after stroke: can perfusion-weighted MRI show functional inactivation? J Cereb Blood Flow Metab 31:1493-1500

15. Liu Y, Karonen JO, Nuutinen J, Vanninen E, Kuikka JT, Vanninen RL (2007) Crossed cerebellar diaschisis in acute ischemic stroke: a study with serial SPECT and MRI. J Cereb Blood Flow Metab 27: 1724-1732

16. Chen S, Guan M, Lian HJ, Ma LJ, Shang JK, He S, Ma MM, Zhang ML, Li ZY, Wang MY, Shi DP, Zhang JW (2014) Crossed cerebellar diaschisis detected by arterial spin-labeled perfusion magnetic resonance imaging in subacute ischemic stroke. J Stroke Cerebrovasc Dis 23:2378-2383

17. Pantano P, Baron JC, Samson Y et al (1986) Crossed cerebellar diaschisis. Further studies. Brain 109:677-694

18. Meyer JS, Obara K, Muramatsu K (1993) Diaschisis. Neurol Res 15:362-366

19. Otte A, Roelcke U, von Ammon K, Hausmann O, Maguire RP, Missimer J, Müller-Brand J, Radü EW, Leenders KL (1998) Crossed cerebellar diaschisis and brain tumor biochemistry studied with positron emission tomography,[18 F] fluorodeoxyglucose and [11 C] methionine. J Neurol Sci 156:73-77

20. Kang KM, Sohn CH, Kim BS, Kim YI, Choi SH, Yun TJ, Kim JH, Park SW, Cheon GJ, Han MH (2015) Correlation of asymmetry indices measured by arterial spin-labeling MR imaging and SPECT in patients with crossed cerebellar diaschisis. AJNR Am J Neuroradiol 36:1662-1668

21. Förster A, Kerl HU, Goerlitz J, Wenz H, Groden C (2014) Crossed cerebellar diaschisis in acute isolated thalamic infarction detected by dynamic susceptibility contrast perfusion MRI. PLoS One 9: e88044

22. Noguchi T, Nishihara M, Egashira Y, Azama S, Hirai T, Kitano I, Yakushiji Y, Kawashima M, Irie H (2015) Arterial spin-labeling MR imaging of cerebral hemorrhages. Neuroradiology 57:1135-1144

23. Kang KM, Sohn CH, Choi SH, Jung KH, Yoo RE, Yun TJ, Kim JH, Park SW (2017) Detection of crossed cerebellar diaschisis in hyperacute ischemic stroke using arterial spin-labeled MR imaging. PLoS One 12(3):e0173971

24. Nocuń A, Wojczal J, Szczepańska-Szerej H, Wilczyński M, Chrapko B (2013) Quantitative evaluation of crossed cerebellar diaschisis, using voxel-based analysis of Tc-99m ECD brain SPECT. Nucl Med Rev Cent East Eur 16:31-34

25. Kim J, Lee SK, Lee JD, Kim YW, Kim DI (2005) Decreased fractional anisotropy of middle cerebellar peduncle in crossed cerebellar diaschisis: diffusion-tensor imaging-positronemission tomography correlation study. AJNR Am J Neuroradiol 26:2224-2228

26. Gold L, Lauritzen M (2002) Neuronal deactivation explains decreased cerebellar blood flow in response to focal cerebral ischemia or suppressed neocortical function. Proc Natl Acad Sci U S A 99: 7699-7704 